\title{
Variable- versus constant-frequency sacral neuromodulation in black-zone overactive bladder patients: a study protocol for a multicenter, prospective, randomized, blind, self-controlled trial
}

\author{
Lingfeng Meng", Zijian Tian", Tongxiang Diao, Miao Wang, Xiaodong Liu, Wei Zhang, Jianye Wang, \\ Yaoguang Zhang \\ Department of Urology, Beijing Hospital, National Center of Gerontology, Institute of Geriatric Medicine, Chinese Academy of Medical Sciences, \\ Beijing, China \\ \#These authors contributed equally to this work. \\ Correspondence to: Yaoguang Zhang. Department of Urology, Beijing Hospital, National Center of Gerontology, Institute of Geriatric Medicine, \\ Chinese Academy of Medical Sciences, No. 1 Dongdan Dahua Road, Dongcheng District, Beijing 100730, China. Email: zhang003887@sina.com.
}

Background: The curative effect of sacral neuromodulation (SNM), used to treat overactive bladder (OAB) patients, is definite. However, some patients still have recurrent symptoms after SNM and unsatisfactory symptom improvement after repeated adjustments of the stimulation parameters combined with oral drugs. These are referred to as black-zone OAB patients. The described SNM is the standard method, which involves a constant-frequency stimulation (CFS) of the patient's specific sacral nerve. A new treatment strategy, which combines the advantages of high-frequency and low-frequency stimulations to generate variable-frequency stimulation (VFS), has not yet undergone a formal randomized clinical trial. Therefore, we designed this clinical trial to evaluate the efficacy and safety of VFS-SNM and CFS-SNM in the treatment of black-zone OAB patients.

Methods: We designed a multicenter, prospective, randomized, blinded, self-controlled trial with a 12week follow-up period. The trial randomly divides the enrolled patients into CFS-SNM and VFS-SNM groups. The main evaluation index is the comparative effectiveness of VFS-SNM and CFS-SNM at the last follow-up. The secondary evaluation indices include the change in the OAB symptom score, the quality of life (QOL) score, and the visual analog scale compared with the baseline period at each follow-up time point. Discussion: Previous studies and our pre-experimental results suggest that black-zone OAB patients may benefit from VFS-SNM. Twelve weeks of VFS-SNM are effective in 40\%, and the non-inferior cutoff value is $10 \%$ ( $80 \%$ power, 0.05 significance level, $20 \%$ loss to follow up). Thus, the calculated sample size is 37 cases each for CFS and VFS groups. The trial is expected to be carried out in 18 centers, but centers will be added or removed as appropriate depending on specific implementation conditions. Clinical researchers at each center will be responsible for screening qualified participants. This is the first randomized controlled trial to comprehensively evaluate the efficacy and safety of VFS-SNM in black-zone OAB patients, which will provide high-quality clinical evidence and may provide new clinical options for such patients.

Trial registration: Chinese Clinical Trial Registry: ChiCTR2000036677, registration date: 24 August 2020 .

Keywords: Sacral neuromodulation (SNM); variable-frequency stimulation (VFS); constant-frequency stimulation (CFS); overactive bladder (OAB)

Submitted Sep 10, 2020. Accepted for publication Dec 10, 2020.

doi: $10.21037 /$ tau-20-1257

View this article at: http://dx.doi.org/10.21037/tau-20-1257 


\section{Introduction}

Overactive bladder $(\mathrm{OAB})$ is a common urinary disease characterized by frequent and urgent urination. The International Continence Society (ICS) defines OAB as a syndrome characterized by urgent urination, often with frequency and nocturnal urination, with or without urgent urinary incontinence (1). The incidence of $\mathrm{OAB}$ is relatively high. A survey study of people over 40 years of age from the United States shows OAB prevalences of 26 $33 \%$ and $27-46 \%$ among men and women of various races, respectively (2). In China, the prevalence of $\mathrm{OAB}$ is lower than that in most previous reports. A population-based, cross-sectional field survey by Wang et al. showed that the prevalence of $\mathrm{OAB}$ in Chinese adults is approximately $4.7-6.0 \%$, that in people over 40 years old is $19.9 \%$, and that it increases with age (3). Although the disease is not life threatening, its complex symptoms seriously affect the quality of life (QOL) of patients, thus creating a large social and economic burden.

There are many treatment methods for OAB. The preferred treatment methods include behavioral therapy, such as lifestyle guidance and bladder training, and drug therapy. However, OAB management may be difficult due to ineffective treatment, intolerable side effects, inability of patients to adhere to treatment, or patient requests for a change in treatment (4). The American Urological Association (AUA) defines refractory $\mathrm{OAB}$ as, poor relief of OAB symptoms after a long course of behavioral training, or failure after 6-12 weeks of treatment with an anticholinergic drug (including poor symptom relief or intolerable adverse reactions) (5).

Since the Food and Drug Administration (FDA) approved sacral neuromodulation (SNM) for the treatment of lower urinary tract dysfunction in 1997, SNM therapy has been increasingly used for patients with refractory $\mathrm{OAB}$, with satisfactory results (6). However, a considerable proportion of patients continue to have recurrent symptoms after SNM, or their degree of symptom improvement weakens with time. Previously, achievement of satisfactory results for these patients was attempted by combining oral drugs and adjusting the frequency, intensity, and time of stimulation. However, there is still a small number of patients (the black-zone patients) in whom the symptomatic improvement remains unsatisfactory.

At present, SNM usually uses a fixed stimulation frequency, that is, the doctor adjusts the stimulation frequency, pulse width, and amplitude, and carries out a single-frequency, single-pulse width and single-amplitude electrical stimulation to the specific sacral nerve of the patient. This is called constant-frequency stimulation (CFS), and it is the current standard SNM method. Previous studies have reported that high-frequency electrical stimulation of the sacral nerve can significantly improve chronic intractable pain in patients (7). On the basis of the correlation between stimulation frequency and the regulation of human motion rhythm, the National Engineering Laboratory of Neuromodulation of Tsinghua University has proposed a variable frequency stimulation (VFS) therapy, which can be used for frequency conversion alternating electrical stimulation according to the patients' clinical symptoms, and automatically changes the frequency in the process of stimulation. In 2015, it was first applied to subthalamic nucleus-deep brain stimulation (STNDBS) to treat Parkinson's disease patients. The results of the study showed that VFS can improve the clinical symptoms of patients more effectively than CFS (8). This inspired the idea and hypotheses for our research because, to our knowledge, no prospective study has compared VFS-SNM with CFS-SNM in black-zone OAB patients. Therefore, our team designed this multicenter, prospective, randomized, blinded, self-controlled trial to evaluate the clinical efficacy of VFS-SNM in black-zone OAB patients, with the aim of providing alternative treatments for $\mathrm{OAB}$ patients. We present the following article in accordance with the SPIRIT reporting checklist (available at http:// dx.doi.org/10.21037/tau-20-1257).

\section{Aims}

First, we plan to evaluate the short-term effects of VFSSNM and CFS-SNM in black-zone OAB patients. Second, we hope to gain insight into whether VFS is more effective than CFS after 12 weeks of treatment.

\section{Methods}

\section{Trial design}

This trial adopts a multicenter, prospective, randomized blind self-control method, and is carried out in designated clinical trial institutions. The research phases of this study are divided into screening/baseline period, pulse generator upgrade, and follow-up period (12 weeks). As shown in the trial flow chart (Figure 1), all participants shut down the device for 7 days during the screening/baseline period, and 


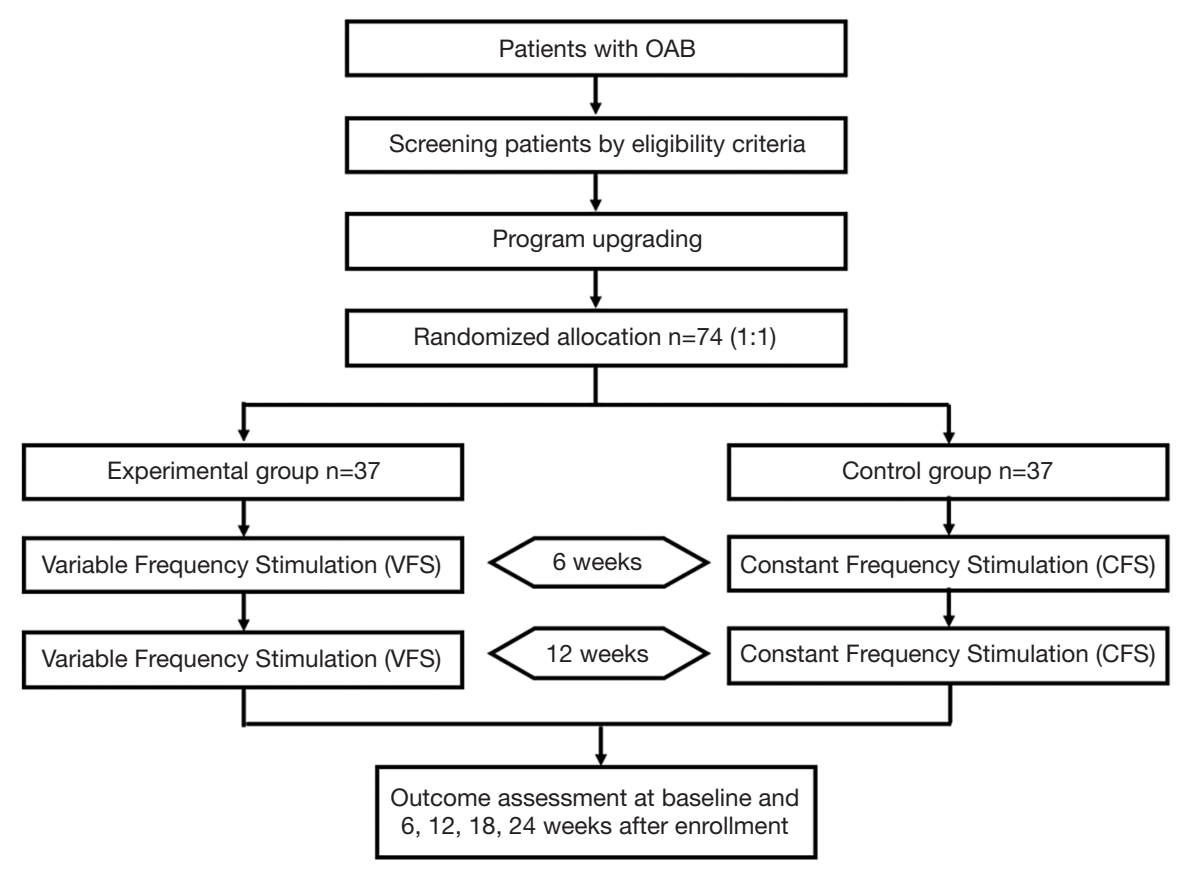

Figure 1 Flow diagram of study participants. OAB, overactive bladder.

then undergo assessment. Those who meet the selection and exclusion criteria sign an informed consent form. After that, the pulse generators of all the selected participants are upgraded to have both the VFS and CFS functions. Then, program-controlled debugging is carried out and programcontrolled parameters are recorded.

According to the ratio of $1: 1$, participants are randomly divided into the VFS and CFS treatment groups. Participants who are randomized to the VFS treatment group are subjected to the VFS parameter setting; while participants who are randomized to the CFS treatment group are subjected to the CFS parameter setting. Safety and effectiveness are evaluated at 6 and 12 weeks after the completion of the setting.

As shown in Table 1, a total of four follow-up surveys are planned. The protocol design is based on the "Consolidated Standards of Reporting Trials and Standard Protocol Items: Recommendations for Interventional Trials (SPIRIT)", and the results of the trial will also be reported according to these guidelines (9). All research procedures were conducted in accordance with the Declaration of Helsinki (as revised in 2013). This study was approved by the Ethics Committee of Beijing Hospital (2020BJYYEC-087-02). Informed consent will be obtained from all participants in accordance with the policies of the board.

\section{Trial center}

Data accrual began March $1^{\text {st }}, 2020$. The trial will be carried out in 18 centers: Beijing Hospital, Peking University First Hospital, Peking University Third Hospital, Peking University People's Hospital, Huadong Hospital Affiliated to Fudan University, Union Hospital Affiliated to Tongji Medical College of Huazhong University of Science and Technology, Second Affiliated Hospital of Nanjing Medical University, Qilu Hospital of Shandong University, Shandong Provincial Hospital, Beijing Chaoyang Hospital, West China Hospital of Sichuan University, Second Hospital Affiliated to Tianjin Medical University, First Hospital of Zhejiang Province, First Affiliated Hospital of Zhengzhou University, China Rehabilitation Research Center, Wuhan General Hospital of Guangzhou Military Region, Southern Theater General Hospital, and Sun Yat-sen Memorial Hospital. However, it is not limited to the above-mentioned centers, and centers will be added or removed as appropriate, depending on the specific implementation conditions. Clinical researchers in each center will be responsible for screening qualified participants.

\section{Inclusion criteria}

Patients who meet the following criteria could be included: 
Table 1 Schedule of recruitment, interventions, and assessments

\begin{tabular}{|c|c|c|c|c|}
\hline Schedule & \multicolumn{2}{|c|}{ Baseline } & \multicolumn{2}{|c|}{ Visit } \\
\hline Time & $3 \pm 2$ days & $5 \pm 2$ days & $40 \pm 14$ days & $90 \pm 14$ days \\
\hline Follow-up & V1 & V2 & V3 & V4 \\
\hline Informed consent & $\mathrm{X}$ & & & \\
\hline Criteria for exclusion & $x$ & & & \\
\hline Medical history/demographic data & $x$ & & & \\
\hline Physical examination & $\mathrm{X}$ & & & \\
\hline Records of side effects & $\mathrm{X}$ & $\mathrm{X}$ & $\mathrm{X}$ & $\mathrm{X}$ \\
\hline Satisfaction survey & & & $\mathrm{x}$ & $\mathrm{x}$ \\
\hline Voiding diary & $x$ & & $\mathrm{x}$ & $x$ \\
\hline Medication & $\mathrm{X}$ & & $\mathrm{X}$ & $\mathrm{X}$ \\
\hline Upgrade to dual mode & & $\mathrm{X}$ & & \\
\hline Random grouping & & $x$ & & \\
\hline Programming & $\mathrm{x}$ & $\mathrm{x}$ & $\mathrm{x}$ & $\mathrm{x}$ \\
\hline Admission confirmed & $\mathrm{x}$ & & & \\
\hline $\mathrm{AE}$ & & $x$ & $\mathrm{x}$ & $\mathrm{x}$ \\
\hline
\end{tabular}

AE, adverse event; CFS, constant-frequency stimulation; CG, control group; EG, experimental group; OABSS, overactive bladder symptom score; QOL, quality of life score; SAE, serious adverse event; VFS, variable-frequency stimulation.

(I) SNM has been performed; (II) patient age $\geq 18$ years; (III) patients with $\mathrm{OAB}$ symptoms, such as urgent urinary incontinence, frequent urination, and urgency, who are non-responsive or unable to tolerate conservative treatment; (IV) after entering the trial, they agree not to increase or decrease the drugs that may affect the lower urinary tract symptoms during the trial; $(\mathrm{V})$ volunteer to participate in this clinical trial, and are able to sign the informed consent; and (VI) able to communicate with the researchers and comply with the trial requirements.

\section{Exclusion criteria}

These include the following: (I) women who are pregnant or breastfeeding; (II) presence of urinary tract infection; (III) organic bladder outlet obstruction; (IV) urinary tumor;
(V) mental illness; (VI) concomitant diseases that seriously affect overall health; (VII) treatment with botulinum toxin in the past 12 months; (VIII) pelvic organ prolapse; and (IX) participation in other clinical trials in the 3 months preceding this trial.

\section{Sample size calculation}

The sample size calculated in this study was determined according to the 12-week treatment effective rate index. The study adopts a non-inferiority design, $\alpha=0.025$, one-sided test; test efficacy $1-\beta=0.8$, and the proportion of the VFS and CFS groups was 1:1. According to the results of previous clinical trials, the effective rate of average daily micturition for 3 months in the CFS group was 37\% (10). Our preexperimental results suggest that after 6 weeks of stimulation, 
the effective rate of VFS is slightly higher than that of CFS.

Therefore, assuming that the effective rate of VFS treatment for 12 weeks is $40 \%$, and the non-inferior cutoff value is $10 \%$, the calculated sample size is 29 cases each for the CFS and VFS groups. We estimated that $20 \%$ of patients would drop out of the study, so the sample size was enlarged to 37 cases each for the CFS and VFS groups. The sample size was calculated based on the following formula:

$$
n_{V}=n_{C}=\frac{\left(Z_{1-\beta}+Z_{1-\alpha}\right)^{2}}{(\delta-\Delta)^{2}}\left[\pi_{C}\left(1-\pi_{C}\right)+\frac{\pi_{V}\left(1-\pi_{V}\right)}{r}\right]
$$

$n_{V}$ : Sample size of the VFS group; $n_{C}$ : Sample size of the CFS group; $\pi_{V}$ : Effective rate of the VFS group; $\pi_{C}$ : Effective rate of the CFS group; $\delta$ : Difference between the rates of the two groups; $\Delta$ : Non-inferiority cutoff value.

\section{Intervention}

\section{CFS}

The physician adjusts the stimulation frequency parameters (i.e., pulse width and amplitude) to set the electrical stimulation to a constant frequency. The participants receive SNM with a single frequency, pulse width, and amplitude.

\section{Variable-frequency stimulation (VFS)}

The VFS parameters will be selected based on the previous relationship between the stimulation frequency and the regulation of human motion rhythm. The main basis for selecting the frequency is the patient's subjective feeling of stimulation. The stimulation frequency will be set to alternate between high and low frequencies. The specific frequencies are $8,10,14$, and $21 \mathrm{~Hz}$, and the stimulation time at each frequency is $10 \mathrm{~s}$. The pulse width during CFS and VFS remains the same, and the amplitude is equal to or slightly lower than the sensory threshold.

\section{Randomization and blinding}

Eligible participants will be randomly assigned to one of two groups (CFS or VFS group) after completion of baseline measurements. The evidence-based Medicine Center of Beijing Hospital will generate an assignment sequence of 1:1 (random numbers will be computer-generated using IBM SPSS Statistics 26.0 software (IBM Corp., Armonk, NY, USA). The computer-generated random numbers will be used to create participant numbers and group lists, which will be placed in opaque sealed envelopes and sent to various research centers.

Randomized numbering and grouping will be completed by research assistants who are not involved in treatment or evaluation. Additionally, efficacy evaluation and data entry will be performed by research assistants who do not participate in the grouping. All participants and researchers (including investigators, trainers, and statisticians) will not know about the grouping and treatment during the entire treatment period. Additionally, the Data and Security Monitoring Board (DSMB) will regularly monitor the work of the center. The DSMB will regularly monitor the trial center when the first participant enters the trial, when the number of enrolled participants reaches half of the number required by the trial, and at different stages after the end of the trial. The inspectors will conduct inspections at any time based on the progress of the trial.

\section{Trial outcomes}

\section{Primary evaluation index}

The primary evaluation index is the efficacy of VFS versus CFS at the 12th week follow-up (mainly including the objective indices in the voiding diary, such as the daily frequency of urination, and the average voiding volume). Specifically, the t-test will be used to compare the clinical treatment efficiency difference between VFS and CFS at 12 weeks after the participants receive treatment. The $95 \%$ confidence interval (CI) of the clinical treatment efficiency will be calculated. If the lower limit of the $95 \%$ CI of the clinical treatment effective rate difference, at 12 weeks after grouping, is greater than zero, it can be considered that the curative effect of VFS is not inferior to that of CFS.

The main efficacy evaluation is based on the full analysis set (FAS) and the per-protocol set (PPS).

\section{Secondary evaluation index}

(I) At the 6th week follow-up, the treatment efficiency of the objective index in the voiding diary of the VFS group will be compared with that of the CFS group.

(II) At each follow-up time point, the OAB symptom score (OABSS), QOL, and visual analog scale (VAS) scores of the two groups will be compared with the baseline.

\section{Safety assessment}

\section{Adverse events (AEs)}

At any time during the trial, all AEs will be recorded on the AE Record Form. Possible AEs include changes in 
stimulation sensation, pain at the implantation or new site, changes in intestinal function, transient mild electric shock sensation, electrode displacement/breakage, infection, and electromagnetic interference.

When an $\mathrm{AE}$ occurs during the whole clinical trial, the researcher should take necessary measures according to the condition of the participant. For example, the researcher can take no measures, adjust stimulation parameters or temporarily stop stimulation treatment, stop the trial, or use drugs. All AEs should be recorded, detailing the process and treatment results until the $\mathrm{AE}$ is properly resolved or stable, and should be followed-up until a satisfactory medical explanation (including loss to follow-up) can be obtained. Researchers can choose hospitalization, outpatient treatment, follow-up, and other treatment methods, according to the severity of the $\mathrm{AE}$.

\section{Serious adverse events (SAEs)}

Refers to the following AEs during the clinical trial: important medical events such as death, life-threatening AEs, AEs requiring hospitalization, or prolonged hospitalization. If no measures are taken to prevent SAEs, they may cause serious harm to the participants.

In the event of a SAE, the investigator must immediately take necessary measures to protect the safety of the participants.

\section{Data collection and management}

All data in the trial will be recorded on the case report form (CRF), which should be filled in carefully by the trial physician. The questionnaire will be filled out by the participants and physicians. Each participant should complete the CRF as soon as possible after the visit. Researchers should ensure that any observations and findings are properly and completely recorded and that they fill out the CRFs carefully.

After the trial is completed and all the CRFs are completed, the researcher will control the quality of data collection. The Research Assistant/Data Manager will be responsible for typing, cleaning, identifying, encoding, and converting the initial data into the appropriate format required for data analysis.

\section{Handling of abnormal data}

\section{Handling of missing data}

The last-visit-carried-forward method will be used to fill in missing data: the latest data that can be observed will be carried forward to the missing data.

\section{Handling of unreasonable and incorrect data}

In the process of data management, the data in the database will be logically checked, and the researcher will be questioned on any unreasonable or incorrect data through a query. The unreasonable or incorrect data will then be adjusted according to the researcher's written replies. The database cannot be locked until all abnormal data are corrected.

\section{Selection criteria and reasons for the participants included in the analysis}

Effectiveness analysis will be conducted based on the FAS and PPS; all pretreatment demographic data analysis will be conducted on the basis of the FAS; and safety evaluation will be conducted based on the safety set (SS).

FAS: the set of participants determined in accordance with the principle of intention to treat refers to the data set composed of all participants who participate in the trial, are implanted with the SNM device, and have pretreatment scores.

PPS: refers to all participants who complete the trial without violations of the protocol.

SS: refers to all participants who participate in the trial, use the SNM, and have at least one safety evaluation.

\section{Statistical analysis}

SPSS Statistics 26.0 will be used for statistical analysis, and the sample size was calculated using PASS 13 software. All statistical tests will be bilateral and $\mathrm{P}<0.05$ will be considered statistically significant.

The comparison of different data will be analyzed by appropriate methods according to the type of data. The intra-group comparison of quantitative data will use the two independent sample data t-test or Wilcoxon rank sum test, according to the data distribution. The chi-square test or exact probability method will be used for classified data. The rank data will be analyzed using the Wilcoxon rank sum test or $\mathrm{CMH}$ test.

\section{Discussion}

$\mathrm{OAB}$ is a syndrome based on clinical symptoms. At present, there is no animal model that can simulate the 
$\mathrm{OAB}$ occurrence. Therefore, current $\mathrm{OAB}$ research is relatively limited, and its mechanism is not clear, which leads to a passive diagnosis and treatment. OAB seriously affects the QOL of patients, and behavioral therapy is recommended as the first-line treatment. If behavioral therapy fails, second-line treatment can be considered; i.e., oral medications, including anti-muscarinic drugs and $\beta 3$ receptor agonists (11). When the second-line treatment is ineffective, or the side effects are unbearable, both the ICS and AUA recommend SNM as the third-line treatment for OAB (Evidence strength: Grade C) (12,13). Additionally, SNM has been approved by the FDA for the treatment of non-neurogenic OAB.

The rapid development of SNM therapy has increased its popularity in China over the past 4 years (14). However, there are still a considerable proportion of patients with refractory OAB receiving SNM who cannot achieve satisfactory clinical efficacy after repeated programming. The treatment of these black-zone patients remains a thorny problem for urologists. Therefore, many scholars have conducted exploratory studies on different stimulation targets (S1-S4), placement of ipsilateral or bilateral electrodes, and new stimulation modes of SNM therapy (15-17). Additionally, Jia et al. reported that VFS-STN achieved good clinical results in patients with Parkinson's disease (8). In our pre-experiment, VFS-SNM also showed satisfactory improvement of symptoms in black-zone $\mathrm{OAB}$ patients. Therefore, we hypothesized that black-zone OAB patients may benefit from VFS-SNM, thus the design of this clinical study protocol.

This clinical trial protocol enables us to evaluate the effects of VFS-SNM on symptoms and scores in blackzone $\mathrm{OAB}$ patients. The purpose of this study is to evaluate the short-term effects and potential side effects of VFS and CFS on black-zone OAB patients, and to explore the effects of different stimulation frequencies on patients' symptoms. In this study, we will use the data in the voiding diary as the primary outcome index, and subjective scores such as OABSS and VAS as the secondary outcome index. To make the data more available, we will recommend that patients record voiding diaries for 3 days, to more accurately reflect the actual voiding status and make the conclusions more reliable.

Nevertheless, the design of this study has certain limitations. The follow-up time of this study is only 12 weeks, and the sample size is small; therefore, the long-term effect of VFS-SNM will remain to be explored. However, this study proposes, for the first time, the application of the
VFS model to SNM. This new model may provide a way to optimize SNM programming and improve the clinical outcomes of black-zone $\mathrm{OAB}$ patients. Additionally, we intend to apply VFS technology to patients who have undergone stage I surgery to determine whether it can change the proportion of patients who choose to undergo stage II surgery. We believe that the research on VFS-SNM will provide new treatment ideas for clinicians and help them choose more suitable treatment methods for patients with various lower urinary tract dysfunction diseases.

\section{Trial registration}

Chinese Clinical Trial Registry: ChiCTR2000036677, registration date: 24 August 2020.

\section{Acknowledgments}

Funding: This work was supported by the National Key R\&D Program of China (grant numbers: 2018YFC2002202, 2016YFC0105505).

\section{Footnote}

Reporting Checklist: The authors have completed the SPIRIT reporting checklist. Available at http://dx.doi.org/10.21037/ tau-20-1257

Conflicts of Interest: All authors have completed the ICMJE uniform disclosure form (available at http://dx.doi. org/10.21037/tau-20-1257). The authors have no conflicts of interest to declare.

Ethical Statement: The authors are accountable for all aspects of the work in ensuring that questions related to the accuracy or integrity of any part of the work are appropriately investigated and resolved. All research procedures were conducted in accordance with the Declaration of Helsinki (as revised in 2013). This study was approved by the Ethics Committee of Beijing Hospital (2020BJYYEC-087-02). Informed consent will be obtained from all participants in accordance with the policies of the board.

Open Access Statement: This is an Open Access article distributed in accordance with the Creative Commons Attribution-NonCommercial-NoDerivs 4.0 International License (CC BY-NC-ND 4.0), which permits the non- 
commercial replication and distribution of the article with the strict proviso that no changes or edits are made and the original work is properly cited (including links to both the formal publication through the relevant DOI and the license). See: https://creativecommons.org/licenses/by-nc-nd/4.0/.

\section{References}

1. Lee UJ, Scott VC, Rashid R, et al. Defining and managing overactive bladder: Disagreement among the experts. Urology 2013;81:257-62.

2. Coyne KS, Margolis MK, Kopp ZS, et al. Racial differences in the prevalence of overactive bladder in the United States from the epidemiology of LUTS (EpiLUTS) study. Urology 2012;79:95-101.

3. Wang $\mathrm{Y}, \mathrm{Xu} \mathrm{K}, \mathrm{Hu} \mathrm{H}$, et al. Prevalence, risk factors, and impact on health related quality of life of overactive bladder in China. Neurourol Urodyn 2011;30:1448-55.

4. Bartley JM, Blum ES, Sirls LT, et al. Understanding clinic options for overactive bladder. Curr Urol Rep 2013;14:541-8.

5. De Wachter S, Benson KD, Dmochowski RR, et al. Sixmonth results of selective bladder denervation in women with refractory overactive bladder. J Urol 2019;201:573-80.

6. Zhang P, Wang JY, Zhang Y, et al. Results of sacral neuromodulation therapy for urinary voiding dysfunction: Five-year experience of a retrospective, multicenter study in China. Neuromodulation 2019;22:730-7.

7. Feler CA, Whitworth LA, Fernandez J. Sacral neuromodulation for chronic pain conditions. Anesthesiol Clin North Am 2003;21:785-95.

8. Jia F, Guo Y, Wan S, et al. Variable frequency stimulation of subthalamic nucleus for freezing of gait in Parkinson's disease. Parkinsonism Relat Disord 2015;21:1471-2.

9. Chan AW, Tetzlaff JM, Altman DG, et al. SPIRIT 2013 statement: Defining standard protocol items for clinical

Cite this article as: Meng L, Tian Z, Diao T, Wang M, Liu X, Zhang W, Wang J, Zhang Y. Variable- versus constantfrequency sacral neuromodulation in black-zone overactive bladder patients: a study protocol for a multicenter, prospective, randomized, blind, self-controlled trial. Transl Androl Urol 2021;10(1):504-511. doi: 10.21037/tau-20-1257 trials. Ann Intern Med 2013;158:200-7.

10. Zhang $\mathrm{Y}$, Zhang $\mathrm{P}$, Tian $\mathrm{X}$, et al. Remotely programmed sacral neuromodulation for the treatment of patients with refractory overactive bladder: A prospective randomized controlled trial evaluating the safety and efficacy of a novel sacral neuromodulation device. World J Urol 2019;37:2481-92.

11. Lightner DJ, Gomelsky A, Souter L, et al. Diagnosis and treatment of overactive bladder (non-neurogenic) in adults: AUA/SUFU guideline amendment 2019. J Urol 2019;202:558-63.

12. Gormley EA, Lightner DJ, Faraday M, et al. Diagnosis and treatment of overactive bladder (non-neurogenic) in adults: AUA/SUFU guideline amendment. J Urol 2015;193:1572-80.

13. Gormley EA, Lightner DJ, Burgio KL, et al. Diagnosis and treatment of overactive bladder (non-neurogenic) in adults: AUA/SUFU guideline. J Urol 2012;188:2455-63.

14. Meng L, Zhang W, Zhang Y, et al. Analysis of the correlation between the clinical effect of sacral neuromodulation and patient age: A retrospective multicenter study in China. Neuromodulation 2020;23:1189-95.

15. Schwalenberg T, Stolzenburg JU, Kriegel C, et al. Sacral neuromodulation in urology - development and current status. Aktuelle Urol 2012;43:39-48.

16. Zabihi N, Mourtzinos A, Maher MG, et al. Short-term results of bilateral S2-S4 sacral neuromodulation for the treatment of refractory interstitial cystitis, painful bladder syndrome, and chronic pelvic pain. Int Urogynecol J Pelvic Floor Dysfunct 2008;19:553-7.

17. Zabihi N, Mourtzinos A, Maher MG, et al. The effects of bilateral caudal epidural S2-4 neuromodulation on female sexual function. Int Urogynecol J Pelvic Floor Dysfunct 2008;19:697-700. 